\title{
Code Development for Simultaneous In-band Transmission of Both SAC-Optical CDMA and WDM Channels
}

\author{
Isaac A. M. Ashour ${ }^{1}$, Student Member, IEEE, Hossam M. H. Shalaby ${ }^{2}$, Senior Member,IEEE and P Susthitha \\ Menon $^{1}$, Member, IEEE \\ ${ }^{1}$ Institute of Microengineering and Nanoelectronics(IMEN), Universiti Kebangsaan Malaysia, 43600 UKM Bangi, \\ Selangor, Malaysia \\ ${ }^{2}$ Department of Electronics and Communications Engineering, School of Electronics, Communications, and \\ Computer Engineering, Egypt-Japan University of Science and Technology (E-JUST), Alexandria 21934, Egypt \\ isaacash@eng.ukm.my
}

\begin{abstract}
Code pulses of spectral amplitude coding (SAC) optical code division multiple-access (OCDMA) are overlaid onto a multichannel wavelength division multiplexing (WDM) system. Modified quadratic congruence (MQC) codes are developed as the signature codes for the SAC/OCDMA system to avoid the overlapping between signals of both systems. In addition, simultaneous transmission of both optical CDMA users and WDM users on the same band is investigated using a network simulation. Our results are compared to traditional non-hybrid systems. It is concluded that the proposed hybrid scheme achieves performance that is slightly worse than that for the non-hybrid scheme. Furthermore, it provides enhanced data confidentiality as compared to the scheme with SAC/OCDMA only because WDM signals perform a partial masking over encoded signals.
\end{abstract}

Keywords - Optical fiber communications, optical networks, optical code division multiple access (OCDMA), spectral amplitude coding (SAC), wavelength division multiplexing (WDM), Modified Quadratic Congruence (MQC).

\section{INTRODUCTION}

Nowadays, Optical CDMA systems have always been of interest because these systems provide the users simultaneous and asynchronous access to the network with high security $[1,2]$. OCDMA can also be overlaid onto the existing WDM networks in order to expand the transmission capacity and to increase the level of security [3, 4]. Modified quadratic congruence (MQC) code of spectral amplitude coding (SAC) for OCDMA is an effective code that can suppress the effects of intensity noise. In addition, this scheme yields a significant reduction in the effects of multiple access interference (MAI) [5].

Recently, the hybrid WDM and SAC/OCDMA scheme have been studied in $[6,7]$, where each WDM channel can employ the same set of SAC/OCDMA systems. In [8,9] the transmission of several spectrally phase-encoded OCDMA channels and conventional DWDM channel within the same ITU window has been demonstrated. We have also demonstrated the simulation of hybrid SAC/OCDMA-WDM overlay system using MQC code in $[10,11]$.
In this paper, we develop a MQC code for simultaneous transmission of several OCDMA channels and WDM channels on the same spectrum band without overlapping. A code shifting technique is used to develop the code. Hence, we investigate the feasibility of transmitting both OCDMA users and WDM users in terms of bit-error rates and of eye diagrams, using a simulation for hybrid and non-hybrid systems. In the following section the MQC code development is presented for our proposed system, followed by the simulation setup, then results and discussions. Finally the conclusion of the paper is provided.

\section{MQC CODE DEVELOPMENT}

An MQC code for transmission of both SAC-Optical CDMA and WDM channels simultaneously without overlapping has been developed. MQC code families are $\left(p^{2}+p, p+1,1\right)$, with $p$ as a prime number and the properties of MOC codes are mentioned in [5], where an MQC code is denoted by $(N, w, \lambda)$, where $N$ is the number of codewords, $w$ is the code weight, and $\lambda$ is the cross-correlation. In this code the optimal number of users $K$ equals $p^{2}$. Table I shows some binary code sequences, $\mathrm{S}$ for parameters $p=5$ and $N=30$.

TABLE I

MQC BINARY SEQUENCES, S WITH $p=5$ and $N=30$ S matrix

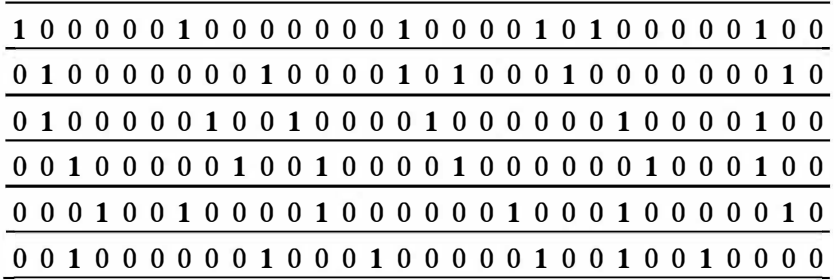

A new binary MQC sequences, $\mathrm{Q}$ is generated based on the old binary MQC sequences, $S$ by using a shifting technique. This technique can be done by multiplication of both $\mathrm{S}$ and $\mathrm{A}_{\mathrm{x}}$ matrix. 
A basic $A_{x}$ is given by $(3 \times 4)$ matrix where $x$ represents the number of $[\mathrm{A}]$ on $\mathrm{A}_{\mathrm{x}}$ and it equals $\frac{N-(N \bmod 3)}{3} \cdot \mathrm{A}_{1}$ and $A_{x}$ are illustrated in equation (1a) and (1b), respectively.

$$
\begin{gathered}
A_{x=1}=\left[\begin{array}{l}
1000 \\
0100 \\
0010
\end{array}\right]_{3 \times 4} \quad(1 \mathrm{a}) \\
A_{x}=\left[\begin{array}{ccccc}
{\left[A_{1}\right]} & 0000 & \cdots & \cdots & \cdots \\
0000 & {\left[A_{2}\right]} & 0000 & \cdots & 0000 \\
0000 & 0000 & \ddots & \cdots & \cdots \\
\vdots & \vdots & & \ddots & \cdots \\
0000 & 0000 & \cdots & 0000 & {\left[A_{x}\right]}
\end{array}\right]_{N \times(N+x)}
\end{gathered}
$$

If $\mathrm{S}$ matrix has size $(K \times N)$, then a size of $\mathrm{A}_{\mathrm{x}}$ is $(N \times M)$ where $M=N+\frac{N-(N \bmod 3)}{3}$, so the result is a new binary code sequences, $\mathrm{Q}$ with a size $(K \times M)$. We notice that the number of columns of $Q$ increases one for each old three columns with consideration of integer number. In our proposed system, we assume that the bandwidth of a WDM channel, $B_{w}$ equals that of a chip of MQC code of SAC/OCDMA, $B_{s}$. That is, $B_{w}=B_{s}=25 \mathrm{GHz}$. The WDM channels are allocated at every $100 \mathrm{GHz}$ (or equivalently 0.8 $\mathrm{nm}$ in wavelength). Therefore, for each 3 elements of MQC chip, there is 1 channel of WDM system. This technique (or method) can be also implemented on any code of SACOCDMA.

In this paper, we present as an example; MQC code sequences with $p=5$ and $K=25$, so the size of $\mathrm{S}$ is $(25 \times$ $30)$. Hence, we select the size of $A_{x}(30 \times 40)$. The result; $Q$ $=\mathrm{S} \times \mathrm{A}_{\mathrm{x}}$ with size of $(25 \times 40)$. This results lead to have 10 locations for the WDM channels as shown in Table II. This technique is important to avoid the overlapping between signals from both systems in order to get a better performance than that presented in our previous work [10, $11]$.

TABLE II

MQC BINARY SEQUENCES WITH SHIFTING PROCESS, Q WITH $p=5, x$ $=10$ and $M=40$

Q matrix

1000000010000000001000000100100000001000 0100000000001000001001000010000000000100


0010000000100010000001000000001000001000 0000100010000010000000001000010000000100 0010000000001000010000000100010001000000

\section{Simulation SETUP FOR THE Proposed SySteM}

In a hybrid WDM-OCDMA system, the network traffic consists of narrow-band WDM signals overlaid with optical broadband CDMA signals in the same spectral region. The main idea in the proposed system is to avoid the overlapping between signals of both systems. By using the code shifting technique that was explained in the above section, the overlapping between signals can be attenuated properly.

The setup of the hybrid scheme, illustrated in Fig. 1, has been simulated using OptiSystem software. The non-hybrid system has also been simulated for comparison with the hybrid system. The MQC code for 25 users used in the simulation is $(30,6,1)$, while the $4 \mathrm{WDM}$ channels used are $1551.4,1550.6,1549.8$ and $1549 \mathrm{~nm}$. The system parameters are illustrated in Table III. The simulation is kept as real as possible by activating all attenuation parameters.

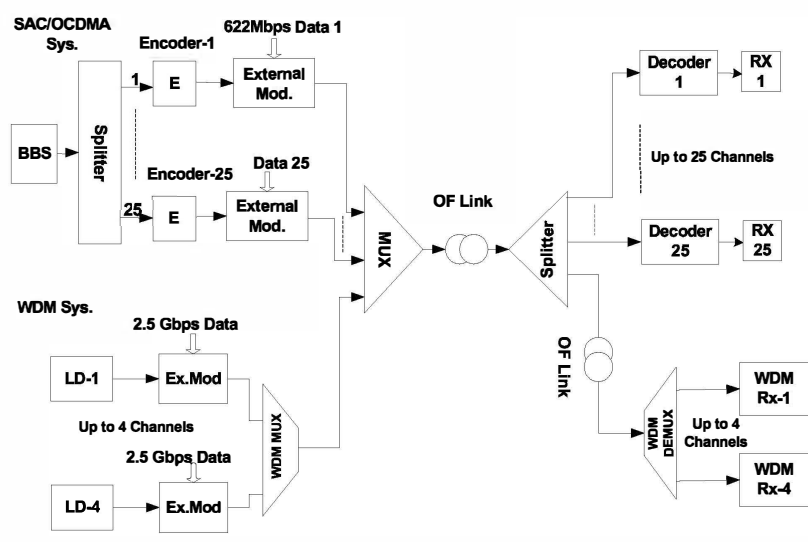

Figure 1. Block-diagram of the hybrid proposed system.

In the hybrid system, the combined signal is transmitted through the optical fiber link and is split into two parts. One part is for the receivers of SAC/OCDMA signals composed of matched decoders, and balance techniques. The other part is for the receivers of the WDM system, composed of narrowband WDM de-multiplexer, to remove the broadband signal that is coming from SAC/OCDMA source, and standard receiver parts of WDM. For the non-hybrid system, each individual system using the same previous parameters were simulated.

TABLE III

TYPICAL PARAMETERS USED FOR EXPERIMENTAL SIMULATION

\begin{tabular}{l|l}
\hline \hline $\begin{array}{l}\text { Broadband source transmitted power for } \\
\text { SAC-OCDMA }\end{array}$ & $16 \mathrm{dBm}$ \\
\hline Laser source transmitted power for WDM & $0 \mathrm{dBm}$ \\
\hline $\begin{array}{l}\text { Data bit rates for SAC/OCDMA and WDM, } \\
\text { respectively }\end{array}$ & $\begin{array}{l}622 \mathrm{Mbps} \text { and } 2.5 \\
\mathrm{Gbps}\end{array}$ \\
\hline Bandwidth of encoder/decoder filters & $25 \mathrm{GHz}$ \\
\hline Fiber link attenuation & $0.2 \mathrm{~dB} / \mathrm{km}$ \\
\hline Fiber dispersion & $17 \mathrm{ps} / \mathrm{nm}-\mathrm{km}$ \\
\hline Dispersion slope & $0.075 \mathrm{ps} / \sqrt{\mathrm{km}}$ \\
\hline External modulator extinction & $30 \mathrm{~dB}$ \\
\hline Dark current & $5 \mathrm{nA}$ \\
\hline $\begin{array}{l}\text { Thermal noise coefficient of the photodetector } \\
\text { for SAC/OCDMA and WDM, respectively }\end{array}$ & $1 \times 10^{-22} \mathrm{~W} / \mathrm{Hz}$ and \\
\hline Number of SAC/OCDMA users & $1.8 \times 10^{-22} \mathrm{~W} / \mathrm{Hz}$ \\
\hline Number of WDM users & 25 \\
\hline \hline
\end{tabular}

\section{RESULTS AND DisCUSSIONS}

The bit-error rate (BER) performance of the system is evaluated prior and after hybridization. Fig. 2 shows the BER for user of SAC-OCDMA system with various received optical power for both hybrid and non-hybrid schemes. The BER for the hybrid scheme is slightly worse than that for the non-hybrid scheme because of the effects of filters. All users 
of SAC-OCDMA system have approximately equivalent BERs. It has been shown that the difference of values

Figure 2. BER versus received optical power for SAC-OCDMA user for both hybrid and non-hybrid systems.

The performance of the WDM system under a hybrid SAC-OCDMA-WDM scheme and lone WDM scheme is shown in Fig. 3 in terms of eye diagrams. These eye diagrams are obtained when a data rate of $2.5 \mathrm{Gbps}$ is transmitted and the received optical power is $-19.0 \mathrm{dBm}$ where Fig. 3(a) is the WDM system in the non-hybrid case and Fig. 3(b) is the WDM system in the hybrid case. The BER is found to be 2.0E-37 for setup as in Fig. 3(a) and 7.9E-35 for setup as in Fig. 3(b). It is obvious that the performance for both systems is near to each other.

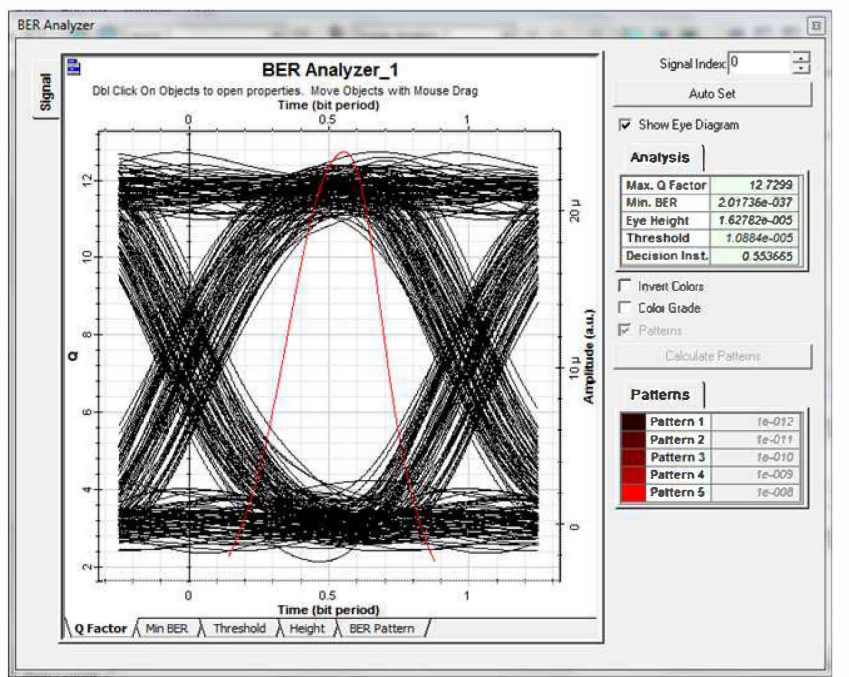

(a)

between the hybrid and non-hybrid system is very small.

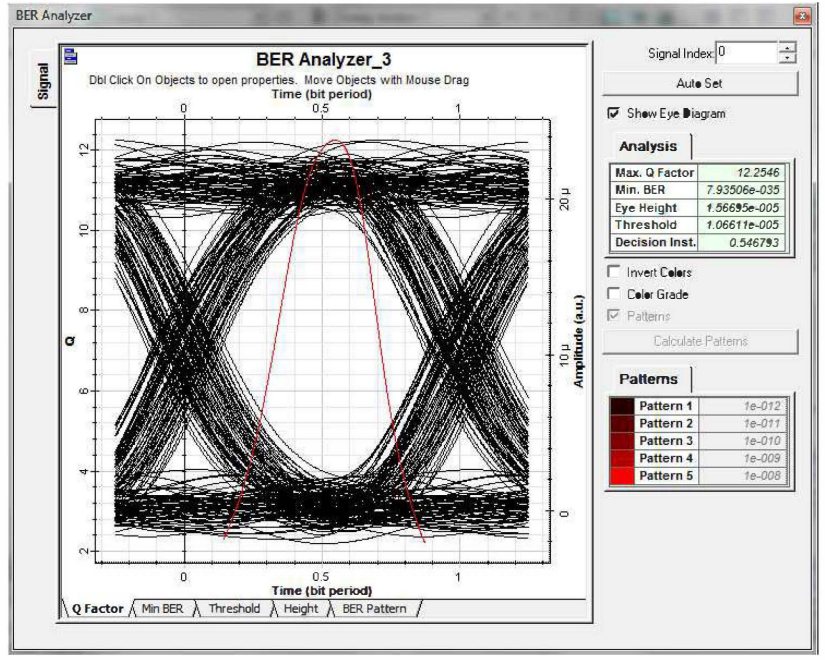

(b)

Figure 3. Eye diagram for a WDM user with $-19.0 \mathrm{dBm}$ received optical power, (a) WDM system; (b) Hybrid SAC-OCDMA-WDM system.

\section{CONCLUSION}

The separation process between different techniques has been presented by developing the MQC code of SAC. The simultaneous in-band transmission of both OCDMA and WDM channels has been investigated. In addition, simulation of both hybrid and non-hybrid schemes have been performed with different design parameters. Our results indicate that although both OCDMA and WDM users use the same band of transmission, an acceptable performance can still be achieved and the difference of values between the hybrid and non-hybrid system is very small. In general, our system provides enhanced data confidentiality as compared to that of SAC/OCDMA scheme only because WDM signals perform a partial masking over encoded signals.

\section{ACKNOWLEDGMENT}

We would like to acknowledge Universiti Kebangsaan Malaysia for sponsoring this project under grant no UKMGGPM-NBT-090-2010 and UKM-OUP-NBT-27-119/2011. We also would like to thank the management of UKM for the fellowship grant "ZAMALAH".

\section{REFERENCES}

[1] J. A. Salehi, "Code division multiple access techniques in optical fiber network-Part I: Fundamental principles," IEEE Trans. Commun., vol. 37, pp. 824-833, Aug. 1989.

[2] J. A. Salehi and C. A. Brackett, "Code division multiple access techniques in optical fiber network-Part II: System performance analysis," IEEE Trans. Commun., vol. 37, pp. 834-842, Aug. 1989.

[3] B.B. Wu, P.R. Prucnal, and E.E. Narimanov, "Secure Transmission Over an Existing Public WDM Lightwave Network," IEEE Photonic Tech. L. 18(17), 1870-1872 (2006). 
[4] B. B. Wu, and E. E. Narimanov, "A method for secure communications over a public fiber-optical network," Opt. Express 14(9), 3738-3751 (2006).

[6] C. C. Yang, "Hybrid Wavelength-Division-Multiplexing/ SpectralAmplitude-Coding Optical CDMA System," IEEE Photonic Tech L. 17(6), 1343-1345 (2005).

[7] J. Huang, Y. Chang, and C. Hsu, "Hybrid WDM and optical CDMA implemented over waveguide-grating-based fiber-to-the-home networks," Opt. Fiber Technol. 13(3), 215-225 (2007).

[8] S. Galli, R. Menendez, P. Toliver, T. Banwell, J. Jackel, J. Young, and S. Etemad, "Experimental results on the simultaneous transmission of two 2.5 Gbps optical-CDMA channels and a $10 \mathrm{Gbps}$ OOK channel within the same WDM window," in Proc. IEEE Optical Fiber Communication (OFC), Anaheim, CA, Mar. 6-11, 2005, vol. 3, pp. 29-31.
[5] Z. Wei, H. M. H. Shalaby, and H. G. Shiraz, "Modified quadratic congruence codes for fiber Bragg-grating-based spectral amplitude coding optical CDMA systems," J. Lightwave Technol. 19(9), 12741281 (2001).

[9] S. Galli, R. Menendez, P. Toliver, T. Banwell, J. Jackel, J. Young, S. Etemad, "Novel Results on the Coexistence of Spectrally Phase Encoded OCDMA and DWDM," submitted to ICC'05, Sep. 2004.

[10] I.A. Ashour, S. Shaari, H.M. Shalaby, and P.S. Menon, "Simulation of MQC code of SAC/Optical CDMA and WDM hybrid overlay system," in Proc. IEEE Conf. International Conference on Modeling, Simulation and Applied Optimization (ICMSAO 2011), pp. 1-4, Kuala Lumpor, Malaysia (2011).

[11] I.A. Ashour, S. Shaari, H.M. Shalaby, P.S. Menon, and H.A. Bakarman, "Hybrid SAC/Optical CDMA-WDM overlay system for enhancing network security," in Proc. IEEE Conf. International Conference on Photonics (ICP2010), pp. 1-4, Langkawi, Malaysia (2010). 